\title{
Incorporation of Ontology-based Technologies and Web 2.0 Mashups in Cloud Com- puting Environment
}

\author{
I-Ching Hsu, Sin-Fong Lyu \\ National Formosa University \\ Taiwan \\ hsuic@nfu.edu.tw \\ SinFong.Lyu565@gmail.com
}

\begin{abstract}
The rapid development of the social Web has resulted in diverse Web 2.0 applications for accessing various publishing information related to social activity such as conference activities, public welfare activities, and cultural events. Due to the masses of social activities information available on the Internet, a major challenge is how to access them efficiently. The traditional keyword search is imprecise and inconvenient for web surfing to find interest information on the internet. This study addresses this issue by defining a Multi-layered Cloud Service Framework (MCSF) for integrating ontology and Web 2.0 technologies into cloud computing environment. The proposed MCSF is used to develop an Intelligent Social Activity Cloud Platform (ISACP) that provides customized social activity for personal needs. To demonstrate the feasibility of ISACP, the experimental results illustrate the efficiency and effectiveness of the proposed approach.
\end{abstract}

Keywords: Cloud Computing, Semantic Web, Web 2.0, Ontology

Received: 26 October 2017, Revised 3 December 2017, Accepted 18 December 2017

DOI: $10.6025 / \mathrm{jcl} / 2018 / 9 / 2 / 81-92$

(C) 2018 DLINE. All Rights Reserved

\section{Introduction}

The popularization of the Internet and the applications of Web 2.0 technologies gave rise to the trend of publishing information related to social activity such as conference activities, public welfare activities, and cultural events on the Internet. The concept of Web 2.0 was proposed by Tim O'Reilly in 2005 [1], with a primary emphasis on resource sharing and user interaction. Web 2.0 is also known as the social web. Mashups [2-4] constitute one of the essential technologies in Web 2.0, integrating multiple web techniques with a wide range of web-related resources with which to develop novel web applications and increase the value of website content. At present, conventional users still search for information by browsing web pages or using keywords in search engines. They must then manually filter through substantial amounts of information and determine whether the information is useful based on their own judgment. This can be extremely time-consuming and inconvenient. The resulting information is generally local, passive, and obtained instantaneously. A lack of intelligent judgment prevents current services from comparing and filtering information quickly. Many Internet services incorporate machine learning or Semantic Web technologies [5-8] to endow systems with intelligent judgment capabilities. They also use user profiles to record and filter information according to the interests of users.

Cloud computing is an emerging business model that has become a popular software development solution in recent years. There

International Journal of Computational Linguistics Research Volume 9 Number 2 June 2018 
are three types of cloud computing service models: Infrastructure as a Service (IaaS), Platform as a Service (PaaS) and Software as a Service (SaaS) [9]. SaaS provides services to cloud clients, while IaaS and PaaS offer services to cloud application developers. In general, the computing applications are developed based on the three different service models. Cloud computing is a service-oriented architecture providing a set of distributed heterogeneous cloud resources delivered via the Internet to cloud computing developers for facilitating the rapid building cloud computing applications. Considering cloud computing from a software engineering perspective, it is a new paradigm for deploying, managing and offering services through a shared infrastructure. Current problems in the development of cloud computing applications are the lack of uniformity in approach to cope up with the heterogeneous effect, including various cloud computing resources, cloud client constraints, and cloud computing middleware. This leads to an inefficient development and a low potential reuse. The Web 2.0 Mashups provide a catalytic solution to this problem.

Within the last few years, the Internet has greatly changed our way of sharing resources and information. As well known, Web 2.0 is recognized as a new generation of web applications proposed by T. O'Reilly. The main feature of Web 2.0 applications is that they provide a medium for the sharing and exchange of cloud resources $[10,11]$. They allow cloud computing developers to take advantage of these cloud resources to reduce the cost or produce new integrated solutions by associating with external resources, which they could not have provided on their own.

Ontology is widely regarded as the best way for knowledge representation and modeling reality because of their semantic modeling ability[12, 13]. At present, many studies [14-16] explore how to use ontology technologies to enhance the intelligence in Web 2.0 applications. However, only a few papers $[17,18]$ study how to facilitate web feeds in intelligent application. This study addresses these issues of social activity information developing an Intelligent Social Activity Cloud Platform (ISACP) based on ontology technologies to describe user preference and annotate social activity to facilitate the automatically generated personalized Web feed.

This study makes three main contributions. First, Web 2.0 Mashup technologies are adopted as a backbone of the Multi-layer Cloud Service Framework (MCSF) to facilitate the interoperability and reusability among heterogeneous social activity information and various cloud service models. Second, the MCSF is adopted to develop a novel ISACP, which serves as a cloud PaaS to allow cloud applications to specify the domain for dynamically intelligent search and filter for personal needs. Third, the ISACP platform can be associated with existing cloud service models, SaaS, PaaS, and IaaS to facilitate the development of intelligent cloud computing applications. Our work integrates three research areas: Cloud Computing, Semantic Web and Web 2.0 .

This paper is organized as follows. The next section presents some of the related work. Section 3 gives an introduction of MCSF. Section 4 presents the architecture of ISACP platform. Section 5 provides a concrete example to illustrate how ontology can be integrated into Hadoop to facilitate intelligent search. The ISACP implementation is presented in section 6, section 7 presents experimental results. Finally, summary and concluding remarks are included in section 8.

\section{Related Work}

The concept of the Semantic Web was proposed by the inventor of the World Wide Web, Tim Berners-Lee, in 1998.. It was proposed to expand the functionality of the web by providing online information with semantic meaning. Such semantics can be used to describe any resources on the Internet, such that even software programs can interpret the Semantic Web to form judgments intelligently. The ability of software programs to process semantically described resources automatically, has made the Semantic Web a mainstream trend in the coming generation of WWW development. The widely-discussed Web 3.0 [19, 20], for example, adopts the Semantic Web to achieve the functions of an intelligent Internet.

Ontology is a philosophical term that describes existing entities and their relationships with one another. However, ontology is also a fundamental component in the Semantic Web [21]. Physically, an ontology is a markup-based file, which is created for a specific domain and used to categorize resources on the Internet into various classes as well as to describe the relationships among them. This achieves the sharing of semantic-bases knowledge and modeling of several aspects of the real world. The languages used to develop ontologies are referred to as ontology languages, of which the recent developments include RDF Schema, SHOE, DAML, DAML+OIL, and OWL. OWL (Web Ontology Language) is the newest ontology language formulated by the World Wide Web Consortium (W3C). 
Cloud computing is an emerging paradigm that provides resources on-demand to cloud consumers through Internet. There are two problems of using cloud computing resources. Cloud resources providers feel difficult to publish their services to cloud users. On the other hand, cloud users cannot easily find useful cloud resources. These problems result from the lack of a uniformly approach to provide intelligence to cope with the interactive effects of cloud computing ecosystem. Our previous research [22] proposed a Semantic Agent as a Service (SAaaS) to integrate agent with semantic web based on cloud computing ecosystem. The main limitation of this platform is that it cannot handle data fusion of Web 2.0 applications. Therefore, this study is dedicated to integrating Web 2.0 and semantic web into cloud computing ecosystem.

Hadoop[23], a cloud computing platform operating primarily on Linux and which is written using Java, is an open source project under the Apache Software Foundation. It provides a distributed computing environment for big data and is specifically used to set up PC clusters. Map Reduce and HDFS (Hadoop Distributed File System) are the two cores of Hadoop [24]; the former enables distributed computing, and the latter achieves a distributed file system with the advantages of high fault tolerance, data localization, simplified parallel computing designs, and easy expansion. Hadoop is suitable for the processing of big data; even with substantial amounts of data, simple hardware expansion will suffice to enhance system efficacy. Map Reduce is a core computing model proposed by Google in 2004. As a parallel programming model, Map Reduce assigns tasks to be processed by clusters comprising thousands of computers. A Map Reduce job generally involves segmenting input data sets, which are then subjected to parallel processing by Map Task. After sorting the data output by Map, the results are input as Reduce tasks. The input and output of the operations are stored in HDFS, which primarily adopts a master-slave framework model. An HDFS cluster comprises a Name Node and multiple Data Nodes. The Name Node is responsible for accessing files and managing the namespace of the file system in the primary server. Internally, the files are divided into multiple data blocks and stored in Data Nodes. A copy mechanism creates backups of the blocks. Using the distributed storage technique, HDFS expands storage space and remote backup capacity.

\section{Multi-layer Cloud Service Framework}

To develop ISACP platform, this section gives an introduction to represent Multi-layer Cloud Service Framework (MCSF), including Physical Machine, HDFS Storage, Algorithm, Application and End User Layers. Figure 1 schematically depicts an illustrative example of Mashup technologies interactions in accordance with the framework, indicating how multi-layer Ontology and MapReduce technologies are adopted.

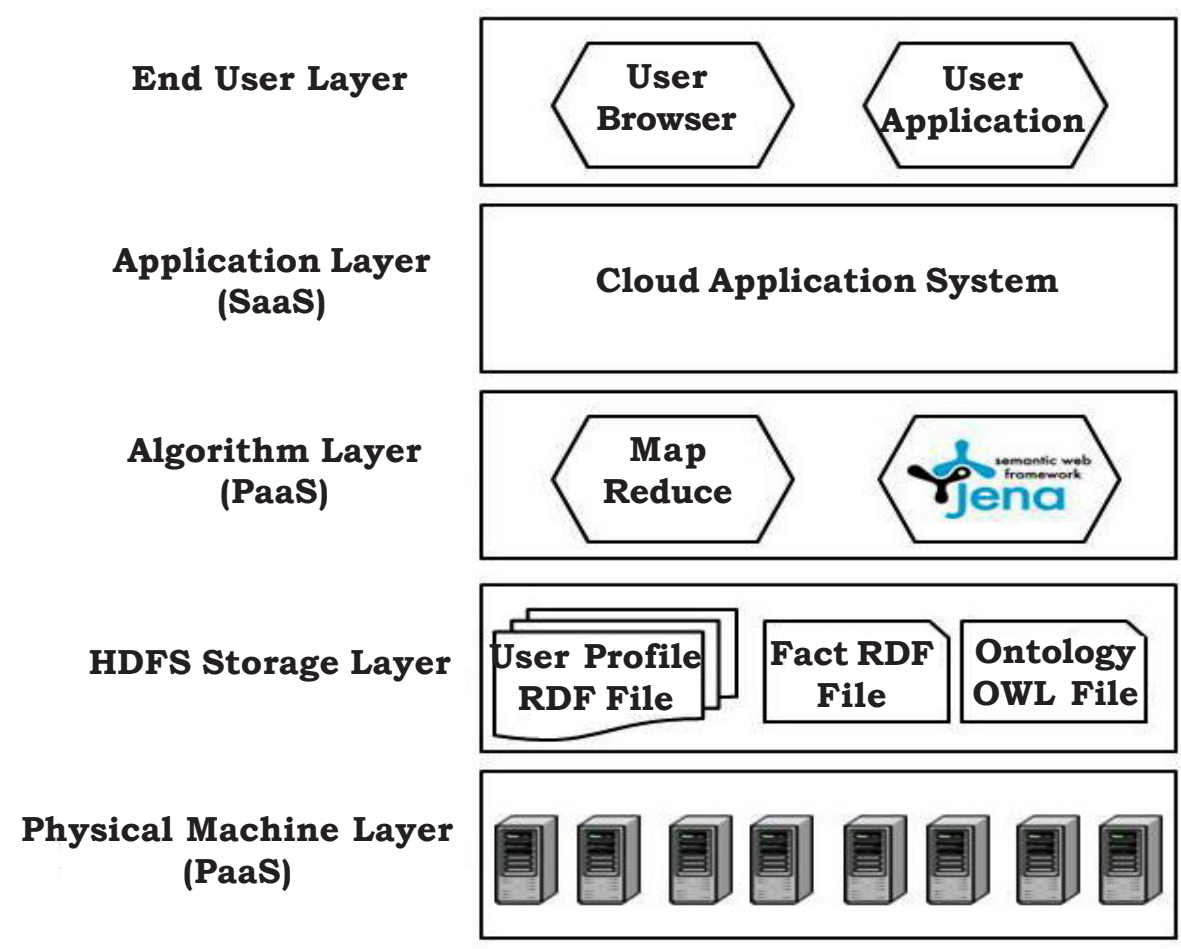

Figure 1. Multi-layer Cloud Service Framework 
The physical machine layer comprises of multiple machines and forms a PC cluster structure via Hadoop. In the event of insufficient computing resources or storage space, rapid computing capabilities and larger storage sspace, we can simply achieve by upgrading the machines or forming links with additional nodes. This provides an IaaS cloud service model. The HDFS storage layer is where the data sets and knowledge bases are stored for Map Reduce computation. The algorithm layer provides a PaaS cloud service model in which the Jena semantics inference engine is incorporated into the Map Reduce program, thereby achieving parallel semantic inference computing. The application layer provides the SaaS cloud service model to make semantic cloud application systems available to various social community domains. This study focuses on seminars, conferences, and speeches associated with information technology. In the end user layer, users can access the cloud application services via browsers or mobile devices.

\section{Intelligent Social activity Cloud Platform}

The Intelligent Social Activity Cloud Platform (ISACP) is developed based on MCSF to associate with the following components, including Hadoop, Web Services, and IoT context client. The dataflow-oriented environment is depicted in Figure 2.

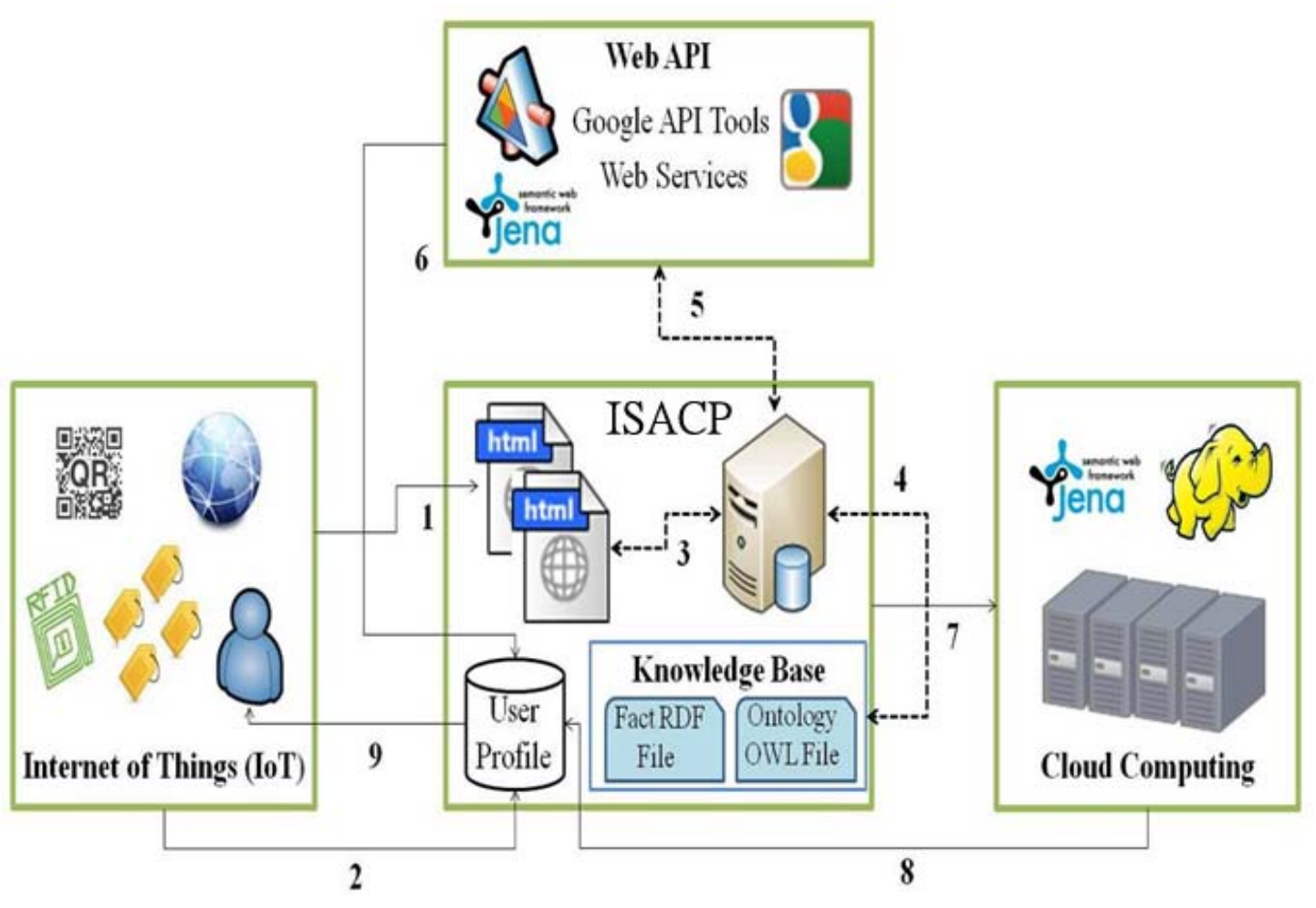

Figure 2. The integrated environment of ISACP

The following steps explain the message flow illustrated in Figure 2

(1) Whether in reality or on the Internet, anyone or anything can be viewed as a part of IOT. The use of web forms or web feed enables users to post information on community events, and IOT concepts can be incorporated to provide the QR codes of the information.

(2) During registration on the platform system, users fill out their personal information and select the community domains that they are interested in. The system then creates a user profile based on the information provided.

(3) The web server provides basic website services, such as posting conference information, making recommendations, and managing tracking lists. 
(4) The knowledge base stores the ontologies and instances of social activity, which are presented and described using the ontology languages RDF(s) and OWL.

(5) The platform contains a Mashup of Web 2.0 applications using Web API to increase the value of website content.

(6) The Jena Web Server features a recommendation function for community events that provides users with information of interest instantaneously.

(7) The size of fact RDF files recording the instances is proportional to the quantity of data. In the event of large quantities of data, the backend Hadoop replaces the instantaneous semantic inference services of Jena to enable substantial semantic inference computing by batch.

(8) Hadoop uses the Map Reduce framework to operate the Jena semantic inference engine for the execution of parallel semantic inference computing. The results are filtered using user profiles before being written and recorded in the RDF files of all user profiles.

(9) Users can easily find information on the events that interest them, as listed in their user profiles.

\section{Ontology integrated with Hadoop-based Cloud Computing Environment}

This study develops an ITC ontology described in Section 5.1. The ontology is used to enhance semantic descriptions for social activity in the specific computer science domain. In section 5.2, we describe how ontology can be integrated into Hadoop-base cloud computing environment.

\subsection{Ontology Development}

Ontologies are used mainly to describe and define online resources. For example, a term may have different meanings in different domains or applications. Among different domains, ontologies present the relations between resources, such as vertical parentchild relationships of horizontal logical relationships, which include a range of characteristics. In our case here the domain is Information Technology Conference (ITC). The ITC ontology is used to capture the semantic-based knowledge in a generic way that provides a commonly agreed structure, such as social activity, information technology, Internet, algorithm, programming, fuzzy, etc., involved in the information technology conference domain. Figure 3 shows the semantic structure of ITC ontology as an UML class diagram. The UML class diagram has as goal to give a graphical overview of the domain concepts and the relations among them. Every entity class and entity property in the diagram has already been described in detail.

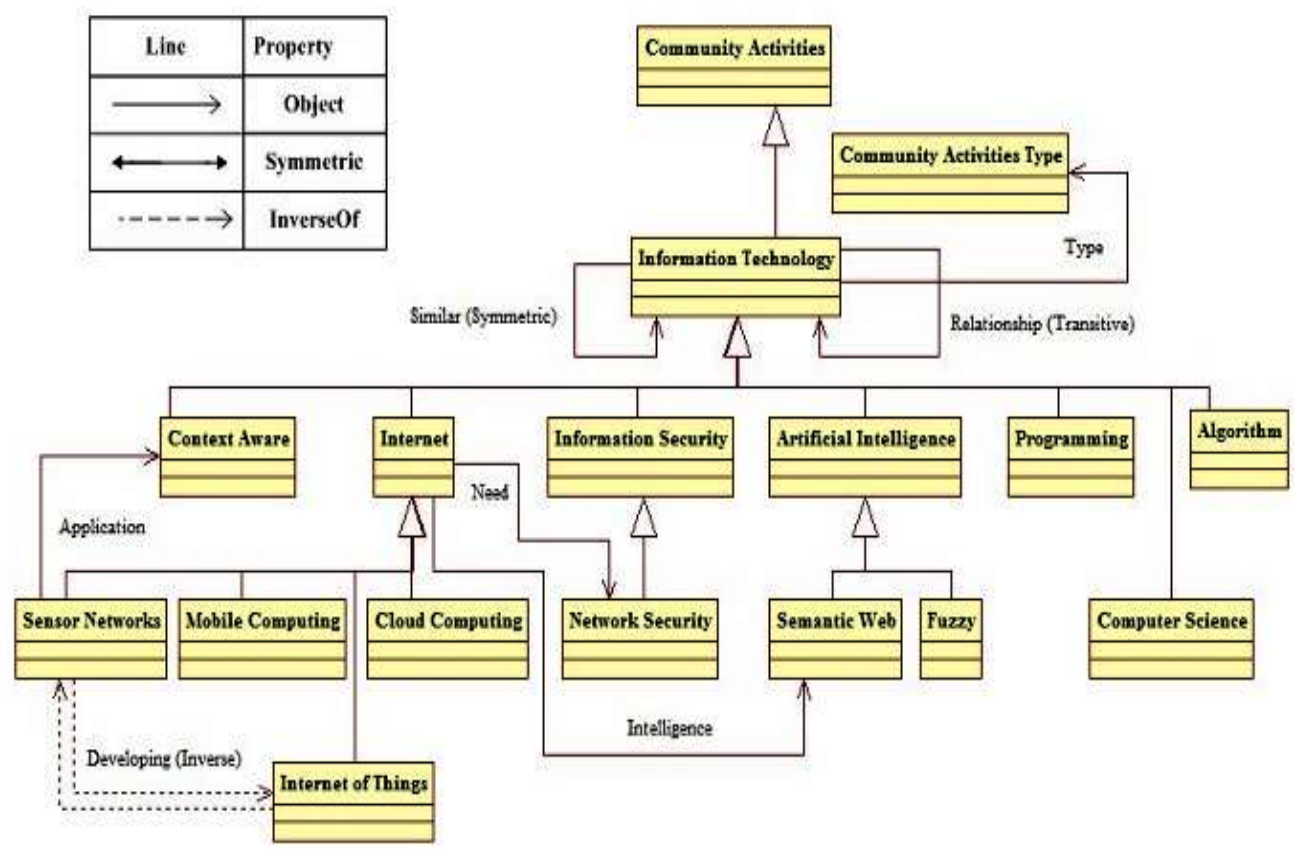

Figure 3. The semantic structure of ITC ontology

International Journal of Computational Linguistics Research Volume 9 Number 2 June 2018 
The following four constraints present some partial code of

The ITC ontology provided OWL-based semantic description, including subclass, inverse property, transitive property, and symmetric property, respectively. A subclass semantic of ITC ontology is showed as follows:

OWL expression

<owl:Class rdf:ID='Information Technology'>

<rdfs: subclassof rdf:resource=' Community Activity' / >

$</$ owl : Class >

Semantic meaning

The Information Technology class is a subclass of the Community Activity class.

Rule expression

if InformationTechnology (x) then CommunityActivity (x)

In addition to the ITC ontology, this study formulated a user profile ontology to describe and record the relevant information and preferred categories of users. The Member class referred to Community Activity class of ITC ontology. Figure 4 presents the user profile ontology using UML.

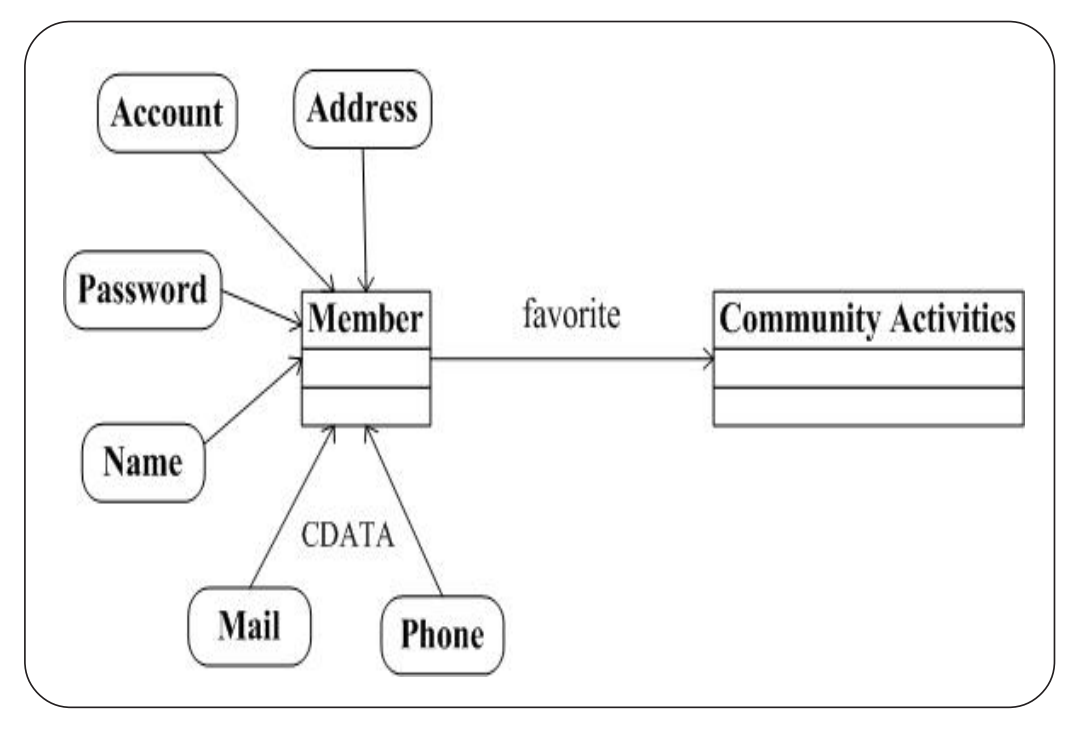

Figure 4. The user profile ontology

\subsection{Integration of Map Reduce with Jena Inference Engine}

Figure 5 presents a diagram of data flow through the Jena semantic inference engine incorporated into the Map Reduce framework. The files subjected to semantic inference (a data set comprising OWL files and Fact RDF files describing the ontologies), are first stored in the HDFS. The Fact RDF files, containing big data, are then divided into several Fact RDF files with smaller data quantities before being subjected to semantic inference using the parallel computing of Map Reduce. The Jena API cannot read the file paths of HDFS protocols in implementation; therefore, we employed the HTTP protocols provided by HDFS to enable the Jena API access to the paths of the OWL files and Fact RDF files in HDFS. Furthermore, we used the document files to record the HTTP URL path of each Fact RDF file in HDFS. The Map Reduce sequence reads the document files to obtain the paths of all segmented Fact RDF files before entering the Map sequence stage. The segmented Fact RDF files provide each Map sequence for semantic inference. Each Map function receives OWL files and segmented Fact RDF files and places them in the Jena semantic inference engine for parallel semantic inference. The results are then sent to Reduce functions and output to the HDFS. 


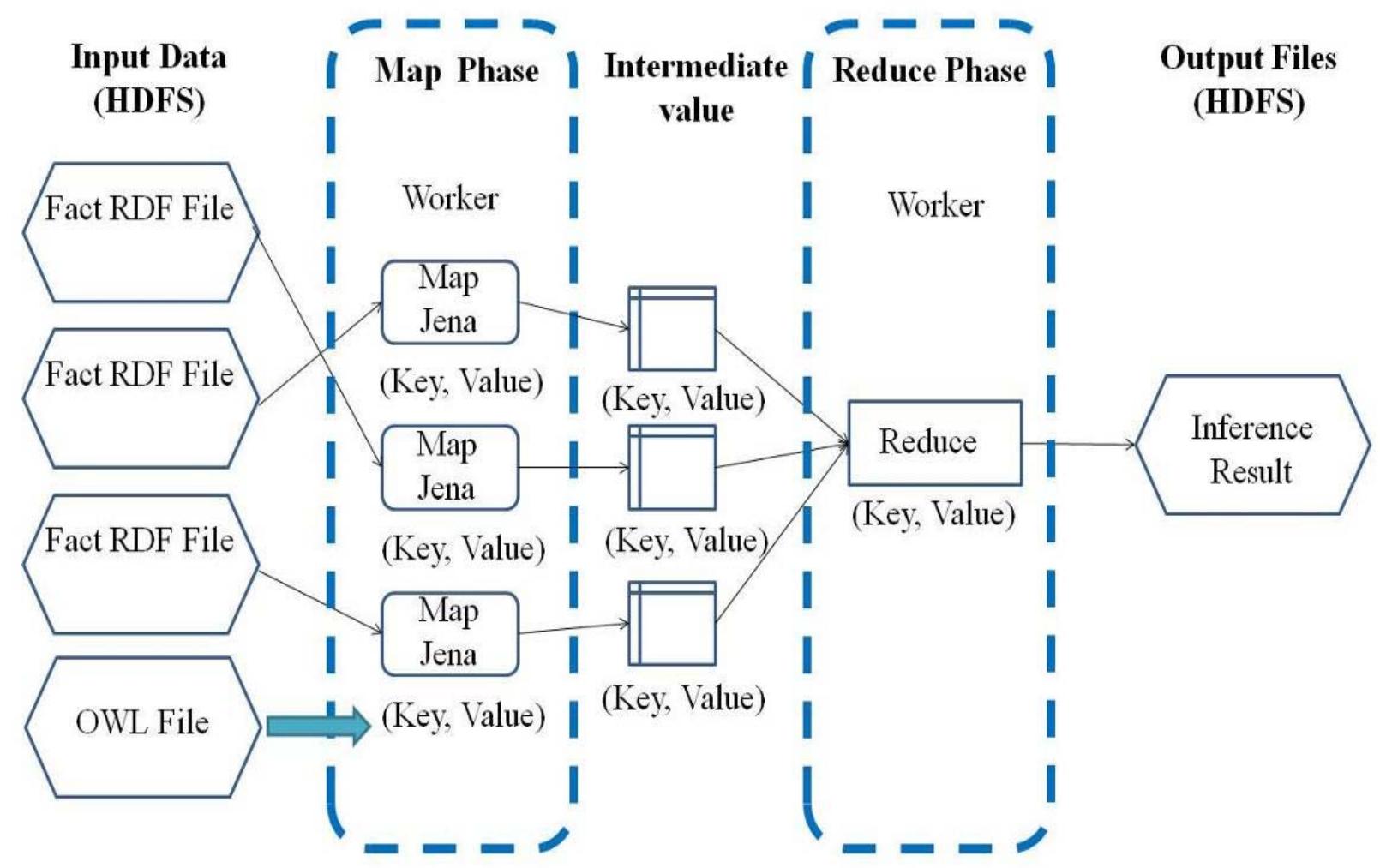

Figure 5. Jena inference engine integrated into MapReduce

\section{ISACP Implementation}

The ISACP can be associated with various domain ontology and metadata. The basic information of social activity is to provide metadata for community members. The ISACP serves as a social activity information broker that supports ontology-based reasoning associated with Hadoop to automatically generate customized information based on personalized needs. The ontology-based reasoning approach provides OWL-based ontologies based on description logics to provide sound and decidable reasoning, including subclass, class intersection, class union, class complement, class disjoint, and class equivalence. Therefore, OWL-based ontologies can enhance the semantic reasoning capabilities of user profiles and social activity information.

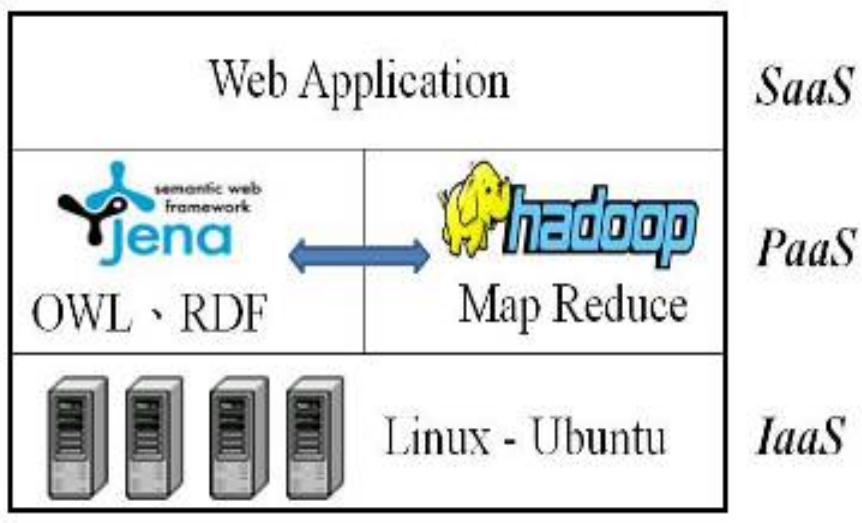

Figure 6. Three type service model of cloud computing environment 
As shown in Figure 6, cloud computing includes three types of service models. The multi-layered cloud service framework founded on Hadoop integrates community activity information, ontology, and Web 2.0 application websites.

As shown in Figure 7, the ISACP enables users or hosting parties to post information on social activity related to IT. Hosting parties are provided an online platform on which they can publicize social activity, thereby increasing exposure. They can also promote cultural and tourism industries near the venue of the event, thereby creating a win-win situation. For users seeking to participate in social activity, the website features a recommendation function integrating large amounts of activity-related information with Semantic Web technologies. Users can also filter through the community activity information according to their preferences, which enables them to reduce the time required for searching information of interest. As present, websites support only the posting of IT community events by schools and academic circles. However, based on the establishment and techniques of the multi-layered cloud service framework, information could be expanded to encompass activities of a similar nature held in a variety of industries or domains, such as technology conferences, product presentations, cultural speeches, tourism exhibitions, and competitions.

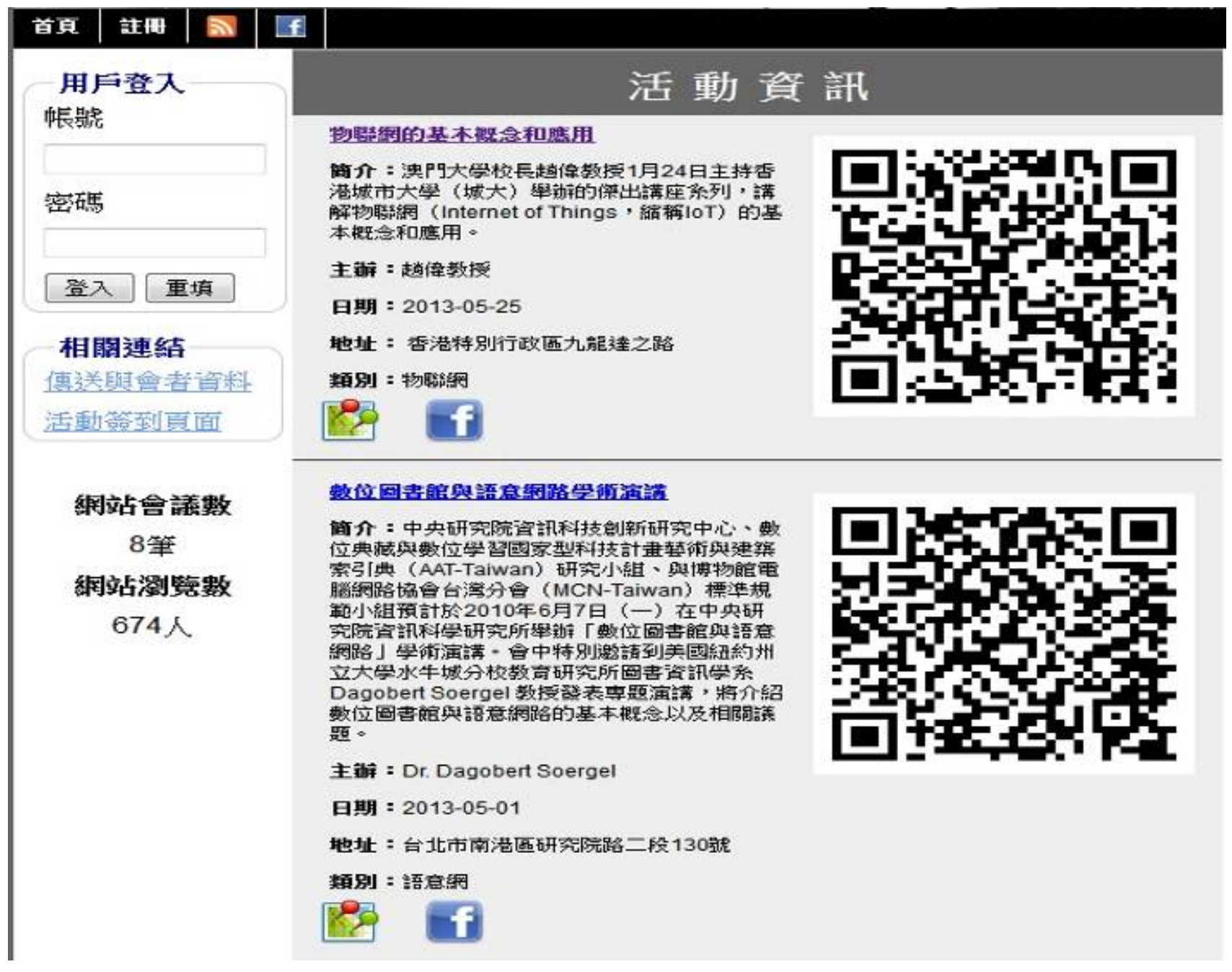

Figure 7. User interface of ISACP

The core technologies of ISACP include the XML Parser Web Service, Jena Web Service, Google API, Mashup, and Hadoop.

\section{- XML Parser Web Service}

In addition to using the webpage form to post information on social activity, the website provides RSS parser service, employing the JDOM Parser Web Feed to post information, in order to enhance the diversity of input information. 


\section{- Jena Web Service}

Instantaneous semantic inference services enable users to filter through information on social activity via their user profile. Users may also receive additional information using the recommendation service provided by the website.

\section{-Hadoop}

The backend Hadoop provides a clustered computing environment and enables large amounts of semantic inference computing by batch through integration with Semantic Web techniques.

\section{- Mashup}

A Mashup of current Web 2.0 applications on the website provides web services and combines Google API Tools, Google Calendar, QR codes, Google Map, and Google Contact to enhance the value of website content.

\section{- Google API Tools}

Google Calendar enables participants to track and schedule social activity, providing instant notification services by e-mail or text message. Google Map allows users to mark the location of community events and plan their routes to the location. Google Contact enables users to recommend social activity to friends on their contact lists as well as send community activity information. The website can generate two types of QR Codes; one enables activity participants to sign in and verify their identification, which saves time for reception staff at events. The other type records the activity information established by the hosting party, such as the contents of event posters.

\section{Experimental Results}

The proposed ISACP has been implemented and described. This section presents an experimental study to evaluate the performance of the ISACP. The performance of ISACP can be evaluated in a local server and in a hadoop-based cluster cloud platform. The experiment had two objectives: the first was to test the performance of the inference mechanism underlying the ontologybased reasoning to determine how ISACP computation time is impacted by quantity of RDF instances. The second objective was to compare the performance of the single server with that of hadoop-based cluster. Additionally, each experiment was repeated 20 times to get the average computation time.

The hadoop-based cluster contains a single name node (the master server) and seven data nodes (the salve server), as shown in Figure 8. The hardware specification of each server is I7-2600 3.4GHz CPU, 16G RAM, and two hard disks with capacities of 2T and

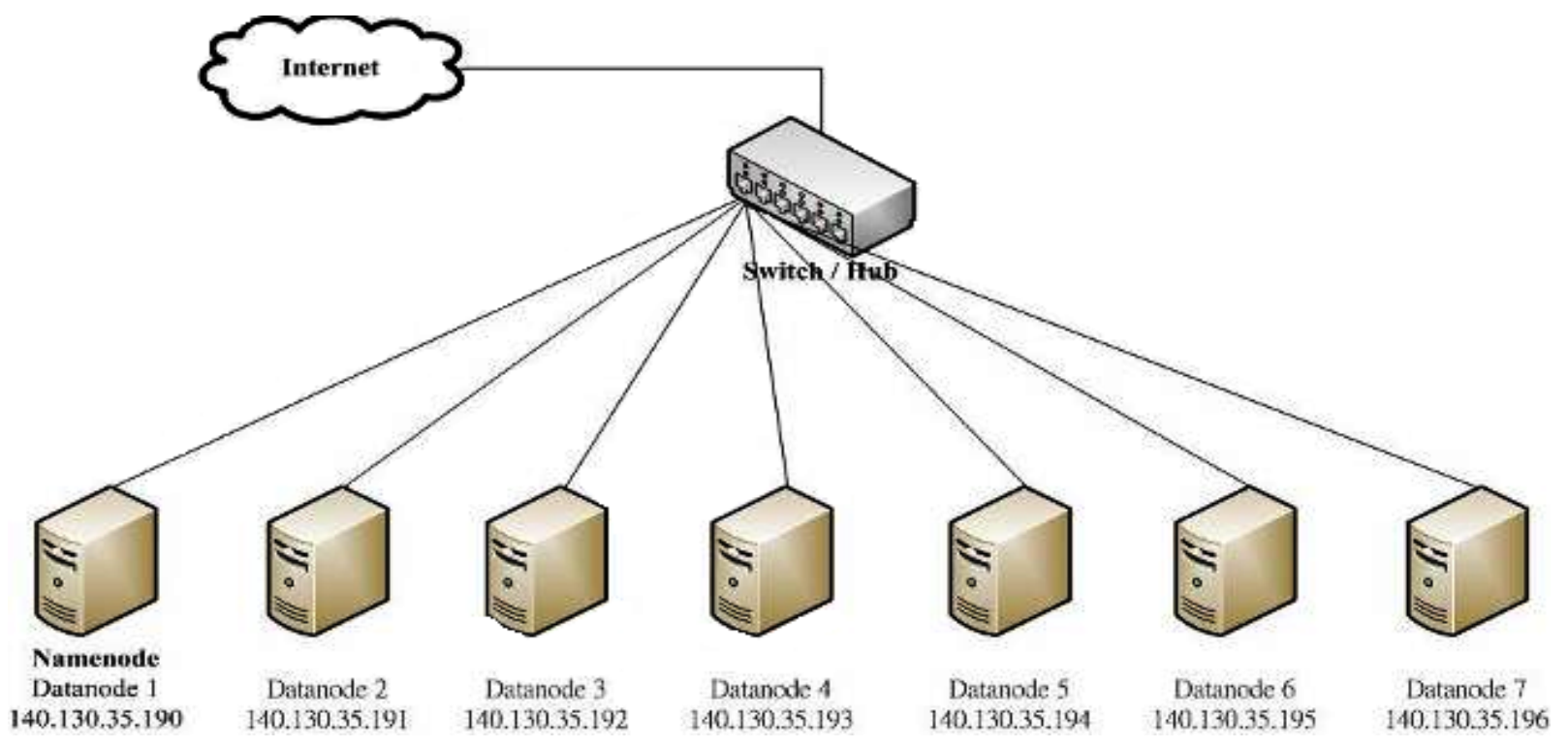

Figure 8. The architecture of hadoop-based cluster 
500G, respectively. The name node manages and stores the file system metadata on a dedicated server. The RDF files are stored on other servers called data nodes. All servers are fully connected and communicate with each other through TCP-based protocols. The performance evaluation is executed based on the different size of RDF file, including 285K, 846K, 1706K, and 6830K. Each file size is evaluated by two approaches, only one Map and five Maps. Table 1 presents the performance of the hadoop-based cluster executing Jena semantic inference.

\begin{tabular}{|l|l|l|}
\hline \multicolumn{1}{|c|}{ Mode } & \multirow{2}{*|}{ 1 Node } & 7 Nodes(cluster) \\
\hline 285k (1 Map) & 36 seconds & $22 \mathrm{~s}$ \\
\hline 285k (5 Maps) & $50 \mathrm{~s}$ & $25 \mathrm{~s}$ \\
\hline 846k (1 Map) & $61 \mathrm{~s}$ & $42 \mathrm{~s}$ \\
\hline 846k (5 Maps) & $54 \mathrm{~s}$ & $28 \mathrm{~s}$ \\
\hline 1706k (1 Map) & $191 \mathrm{~s}$ & $120 \mathrm{~s}$ \\
\hline $\mathbf{1 7 0 6 k}$ (5 Maps) & $57 \mathrm{~s}$ & $30 \mathrm{~s}$ \\
\hline $\mathbf{6 8 3 0 k}$ (1 Map) & $1800 \mathrm{~s}$ & $1080 \mathrm{~s}$ \\
\hline $\mathbf{6 8 3 0 k}$ (5 Maps) & 285s & $79 \mathrm{~s}$ \\
\hline
\end{tabular}

Table 1. Test results

The evaluation shows that the hadoop-based cluster is able to efficiently process a large size of RDF file and that it scales linearly regarding the RDF file size and Map number of Hadoop. The results expose that the huge amount of RDF instances can be stored in the proposed hadoop-based cluster, which built mostly by cheap hardware and still can propose fast enough. We conclude that the proposed ISACP is a scalable framework to efficiently propose large amount of RDF instances.

\section{Conclusions}

Posting information related to social activity on the Internet is popular; however, users must undertake the time-consuming and inconvenient task of filtering through the substantial amounts of information or using keywords in search engines to search for information of interest. Such community activity information is local, passive, and instantaneous, and the growing quantity prevents users from obtaining the information they desire quickly. Thus, this study proposed a multi-layered cloud-based service model, incorporating current Web 2.0 technologies and Semantic Web technologies into Hadoop. We then constructed an intelligent community activity cloud platform as well as a web-based intelligent conference cloud website for exhibition and validation.

With the growth in the quantity of information related to social activity, the relationships among this data become increasingly complex. The means of automatically segmenting ontology files and fact files and inserting MapReduce calculations are the focus of this study. In the future, we will incorporate Eucalyptus to enable the capabilities of web hosting platforms and facilitate commercial services and mobile cloud computing [33-35]. We will also work with businesses associated with transportation and accommodation located in the vicinity of social activity in order to provide discounts to attract crowds and promote local industry. Recommendations regarding local fare, specialties, and tourist attractions will also be provided. In addition to information on IT conferences, the platform could also be extended to encompass information on a wide range of activities of a similar nature and Internet of things (IOT) technologies [36].

The main aim of this research was to investigate how Cloud Computing, Semantic Web, and Web 2.0 technologies can be integrated to develop a novel Cloud PaaS, which can offer dynamic intelligent search and filter for cloud computing applications. This aim was tackled by a four steps approach, as articulated in the following objective: 
1. Adopt the Multi-layer Cloud Service Framework (MCSF) to enhance the computer reasoning, and the original Hadoop still exists to cooperate with ontologies and rules.

2. Develop a novel Intelligent Social Activity Cloud Platform (ISACP) based on the MCSF.

3. Demonstrate the potential of ISACP and how an intelligent cloud computing application can be developed associated with ISACP.

4. Implement a prototype system of ISACP that can support computer science domain ontology and knowledge to offer dynamic intelligent search for cloud computing applications.

\section{Acknowledgments}

The authors would like to thank the National Science Council of the Republic of China, Taiwan for financially supporting this research under Contract No. NSC 101-2221-E-150-054 and NSC 102-2221-E-150-061.

\section{References}

[1] O’Reilly, T. (2005, 8 Oct. 2016). What Is Web 2.0. Available: http://www.oreillynet.com/pub/a/oreilly/tim/news/2005/09/30/ what-is-web-20.html

[2] Tsai, C.-C., Lee, C.-J., Tang, S.-M. (2009). The web 2.0 movement: Mashups driven and web services, WSEAS Transactions on Computers, V. 8, p. 235-1244, 2009.

[3] Solomou, G. D., Kalou, A. K., Koutsomitropoulos, D. A., Papatheodorou, T. S. (2011). A Mashup Personalization Service based on Semantic Web Rules and Linked Data, In: 2011 Seventh International Conference on Signal Image Technology \& Internet-Based Systems, Dijon, France.

[4] Hsu, I.-C. (2013). Personalized Web Feeds based on Ontology Technologies, Information Systems Frontiers, 15, p. 465-479.

[5] Hsu, I.-C. (2013). Integrating Ontology Technology with Folksonomies for Personalized Social Tag Recommendation, Applied Soft Computing, 13, p. 3745-3750.

[6] Pai, F.-P., Hsu, I.-C., Chung, Y.-C. (2016). Semantic web technology for agent interoperability: a proposed infrastructure, Applied Intelligence, 44, p. 1-16.

[7] Peng, L. (2016). A semantic web service discovery system based on UDDI and OWL-S, International Journal of u- and eService, Science and Technology, 9, p 187-200.

[8] Soylu, A., Giese, M., Jimenez-Ruiz, E., Kharlamov, E., Zheleznyakov, D., Horrocks, I. (2017). Ontology-based end-user visual query formulation: Why, what, who, how, and which?, Universal Access in the Information Society, 19, p. 435-467.

[9] Mell, P., Grance. T. (2009, 30 Aug. 2011). The NIST Definition of Cloud Computing. Available: http://csrc.nist.gov/groups/SNS/ cloud-computing/index.html

[10] Yakovlev, I. V. (2007). Web 2.0: Is It Evolutionary or Revolutionary? IEEE IT Professional, 9, p 43-45.

[11] Goudas, T., Louizos, C., Petasis, G., Karkaletsis, V. (2015). Argument Extraction from News, Blogs, and the Social Web, International Journal on Artificial Intelligence Tools, 24, 2015.

[13] Salem, Y. B., Idodi, R., Ettabaa, K. S., Hamrouni, K., Solaiman, B. (2017). High level mammographic information fusion for real world ontology population, Journal of Digital Information Management , 15, p 259-271.

[14] Kumar, S., Mastorakis, N. E. (2010). Novel models for multi-agent negotiation based semantic web service composition, WSEAS Transactions on Computers, 9, p. 339-350.

[15] Acuña, C. J., Minoli, M., Marcos, E. (2010). Integrating web portals with semantic web services: A case study, International Journal of Enterprise Information Systems, 6.

[16] García-Silva, A., García-Castro, L. J., García, A., Corcho, O. (2015). Building domain ontologies out of folksonomies and linked data,” International Journal on Artificial Intelligence Tools, 24.

International Journal of Computational Linguistics Research Volume 9 Number 2 June 2018 
[17] Maio, C. D., Fenza, G., Loia, V., Senatore, S. (2010). Ontology-based knowledge structuring: an application on RSS Feeds, presented at the HSI 2009.

[18] MahmoudiNasab, H., Sakr, S. (2009). FeedRank: A Semantic-Based Management System of Web Feeds, In: IDEAL 2009, LNCS 5788, p. 126-133.

[19] Cabage, N., Zhang, S. (2013). Web 3.0 has begun, Interactions, 20, p 26-31.

[20] Lai, H., Fan, Y., Xin, L., Liang, H. (2013). The framework of web 3.0-based enterprise knowledge management system, In: 7th International Conference on Knowledge Management in Organizations: Service andCloud Computing,

[21] Berners-Lee, T., Hendler, J., Lassila, O. (2001). The Semantic Web - A new form of Web content that is meaningful to computers will unleash a revolution of new possibilities, Scientific American, 284, p. 34-43, May 2001.

[22] Hsu, I.-C., Cheng, F.-Q. (2015). SAaaS: a cloud computing service model using semantic-based agent, Expert Systems, 32, p 77-93.

[23] Apache. (Jan. 12, 2012). Apache Hadoop. Available: http://hadoop.apache.org/

[24] Slagter, K., Hsu, C.-H., Chung, Y.-C., Zhang, D. (2013). An improved partitioning mechanism for optimizing massive data analysis using MapReduce, Journal of Supercomputing, 66, p. 539-555.

[25] Park, J., Jeong, H., Jeong, Y.-S., Lee, E. (2014).Two-phase grouping-based resource management for big data processing in mobile cloud computing, International Journal of Communication Systems, 27, p. 839-851.

[26] Shiraz, M., Ahmed, E., Gani, A., Han, Q. (2014). Investigation on runtime partitioning of elastic mobile applications for mobile cloud computing, Journal of Supercomputing, 67, p. 84-103.

[27] Shiraz, M., Gani, A. (2014). A lightweight active service migration framework for computational offloading in mobile cloud computing, Journal of Supercomputing 68, p. 978-995.

[28] Ding, Z., Chen, Z., Yang, Q. (2014). IoT-SVKSearch: A real-time multimodal search engine mechanism for the internet of things, International Journal of Communication Systems, 27, p 871-897. 